\title{
Nonlinear underwater robot controller design with adaptive disturbance prediction and smoother
}

\author{
Xin Song ${ }^{\text {a,b,c }}$, Fang Liu ${ }^{\mathrm{e}}$, ZaoJian Zou ${ }^{\mathrm{b}, \mathrm{d}}$, Yue-Min Zhu ${ }^{\mathrm{c}}$, JianChuan Yin ${ }^{\mathrm{b}}$, Feng Xu ${ }^{\mathrm{b}}$ \\ a China Ship Research and Development Academy, Beijing, 100192, P.R. China \\ ${ }^{\mathrm{b}}$ School of Naval Architecture, Ocean and Civil Engineering, Shanghai Jiao Tong University \\ Shanghai, 200240, P.R.China. \\ ${ }^{\mathrm{c}}$ CREATIS, CNRS UMR 5220, INSERM U630, INSA Lyon, University of Lyon \\ Villeurbanne, 69621, France. \\ d State Key Laboratory of Ocean Engineering, Shanghai Jiao Tong University \\ Shanghai, 200240, P. R. China. \\ ${ }^{e}$ School of humanities, Zhejiang Ocean University, Zhoushan, 316000, P. R. China \\ E-mail:songxsx@gmail.com \\ Received 27 September 2010 \\ Accepted 20 June 2011
}

\begin{abstract}
A new hybrid adaptive control algorithm is proposed for the nonlinear system controller design of underwater robot Compared with the previous works in the controller design of underwater robot, the main advantages of this work are: (1) A new disturbance prediction and compensation model is proposed; (2) A new adaptive fuzzy smoother is proposed for the control input; (3) A time-varying flow disturbance is considered for the control design which is always neglected in many previous works and several practical experiments under different environment were implemented to verify the control performance. The Lyapunov stability theory proves the stability and convergence of this new control system. Simulation and experiment results demonstrate the performance and the effectiveness of this new algorithm.
\end{abstract}

Keywords: adaptive control; fuzzy sliding mode; underwater robot; disturbance prediction; compensation

\section{Introduction}

The nonlinear system controller is always a top and interesting research. Adaptive control of these systems has been paid a lot of attention during these two decades. The controller design of the underwater robot is an important application of the adaptive nonlinear theory. The complex underwater environment always causes troubles. Thus, it is uneasy to control the underwater robot well in practice. The motion of underwater robot is modeled in six degree of freedom (DoF) and the disturbance in the water including the flow, cable etc. is uneasy to be calculated. CFD technology can be applied for the simulation of the underwater robot motion. However, the large time of CFD calculation makes the direct control unavailable. A growing number of researchers have published many papers and dedicated different control methods to the controller of the underwater robot ${ }^{1-5}$. Some comparison studies of several algorithms have been carried out ${ }^{6}$. It has been confirmed that the necessity of the controller, which could deal with the inherent nonlinear system dynamics, uncertain hydrodynamic coefficients and disturbances.

Due to the robustness to system uncertainties, disturbance etc., the sliding mode control is proven to be an attractive approach for underwater robot. However, the chattering phenomenon that exists in the traditional method makes the algorithm improper for the real application. Thus, an approach for eliminating this drawback should be studied. Meanwhile, fuzzy logic 
theory ${ }^{7,8}$ has been largely employed to control and identify the system because of its possibility of expressing the human experience. Some researchers have combined the sliding mode control and fuzzy logic control to realize the adaptive nonlinear controller ${ }^{9-14}$.

Here, in this paper, a new adaptive controller is proposed to control the yaw motion of underwater robot. This proposed controller consists of a new adaptive fuzzy model that is developed to predict and compensate the external disturbances and an adaptive fuzzy smoother that is proposed to improve the control output performance. Using the certification of Lyapunov stability theory and Barbalat's lemma, the stability and convergence properties of this nonlinear control system are proven. A time-varying, large flow disturbance is proposed to be considered in the simulations, which is always ignored or considered as ideal conditions with minor effects in the previous works. However, the disturbance is important and necessary for application of underwater robot. To demonstrate the effectiveness of this new proposed algorithm, several practical experiments have been implemented. It should be mentioned that many previous works seldom apply their algorithms into practical experiments and these works lose the confidences in building the effective control schemes for real underwater robot applications. Through the experiments in different environment, the performance and robustness of this new proposed algorithm are demonstrated. However, there are some shortcomings appeared in the applications. The reasons of these shortcomings are discussed and the future works are pointed out.

The rest of this paper is organized as follows: section 2 shows the underwater robot kinematics and dynamics; section 3 introduces theory of T-S fuzzy model; section 4 proposes the adaptive controller design; section 5 presents and discusses the details of simulations and experiments. The final section provides concluding remarks.

\section{Underwater robot kinematics and dynamics}

The motion of underwater robot is mainly described in two reference systems: one is the earth-fixed frame system, which is assumed to be inertia and the other is the body-fixed frame, which is attached to the underwater robot ${ }^{15}$. Body-fixed frame's axes $\left(X_{B}, Y_{B}, Z_{B}\right)$ are in accordance with the principle axes of inertia. The original point is usually set at the center of gravity. The position of the underwater robot, $\eta_{p}=[x, y, z]^{T}$, and orientation, $\eta_{0}=[\phi, \theta, \psi]^{T}$, (roll angle, pitch angle, yaw angle) are described according to the inertial reference frame. Linear velocity vector $v_{p}=[u, v, w]^{T}$ (sway, surge, heave) and angular velocity vector $v_{p}=[p, q, r]^{T}$ (rate of roll, pitch rate, yaw rate) are expressed with respect to the body-fixed frame. Fig.1. shows all notations.

Generally, the underwater robot involves several dynamic behaviors including the rigid dynamics of vehicle's body, the representation of the surrounding fluid dynamics or the dynamics of the tether cable. Thus, the mathematic model consists of ordinary differential equations, rigid-body dynamics, and partial differential equations that represent fluid dynamics (Navier-stokes equations). To overcome such a complex computation burden, according to Ref. 15, a motion dynamic equation of underwater robot in the body-fixed reference frame can be represented as

$$
\begin{gathered}
M(\eta) \ddot{\eta}+C(\eta, \dot{\eta}) \dot{\eta}+D(\eta, \dot{\eta}) \dot{\eta}+g(\eta)=\tau \\
\dot{\eta}=J(\eta) v .
\end{gathered}
$$

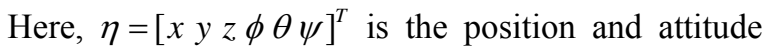
vector with respect to the inertial frame, $M(\eta) \in R^{6 \times 6}$ is the inertial matrix including adding inertial part, $C(\eta, \dot{\eta}) \dot{\eta} \in R^{6}$ is the centripetal and coriolis term, $D(\eta, \dot{\eta}) \dot{\eta} \in R^{6}$ is the damping term, $g(\eta) \in R^{6}$ is the gravity and buoyancy. $J(\eta) \in R^{6 \times 6}$ is the transform matrix between the body-fixed reference and the earthfixed reference, $\tau \in R^{6 \times 1}$ is the control input (thrust force), $v=\left[\begin{array}{llll}u & v & p & q\end{array}\right]^{T}$ is the vector of linear and angle velocity with respect to the body-fixed frame. The underwater robot dynamic behavior can be approximated by many approaches ${ }^{12,16}$.

The dynamics of underwater robot are assumed to have the following characters:

(i) The inertia matrix $M(\eta)$ is symmetric and positive definite;

(ii) Matrix $\dot{M}(\eta)-2 C(\eta, \dot{\eta})$ is skew and symmetric;

(iii) Damping matrix $D(\eta, \dot{\eta})$ is strictly positive and within limited boundary;

(iv) Coriolis matrix is in limited boundary and $\dot{\eta}$ is linear. There is a positive number $k_{c}$ to make

$$
\|C(\eta, \dot{\eta})\| \leq k_{c}\|\dot{\eta}\| .
$$


In this research, the underwater robot motion simulations neglect the wave interference, and only consider the impact of water flow. Water density is considered constant without the sand stone effects. The vertical inclination angle is assumed to be less than $\pi / 2$.

In order to guarantee the feasibility of experimental model, the following assumptions are predefined:

(i) Low velocity of the underwater robots;

(ii) The damping force is non-coupling;

(iii) The linear damping force is negligible;

(iv) Water kinetic coefficient is constant;

In the parts of roll and pitch angle, the distance between buoyancy and gravity center is usually large enough to keep them small and the system is stable. Thus, the hydrodynamic equations in these directions are not necessary to be considered.

Generally, the dynamic parameters of underwater robot are strongly nonlinear, coupled, and uncertain. Some parts of the hydrodynamic model are estimated. The exact values of hydrodynamic coefficients are difficult to be calculated. Comparing with other works of underwater robots, the uncertainties in this research are assumed that they are bounded and can be estimated with this new adaptive fuzzy prediction. Meanwhile, this adaptive controller is applied to reduce the chattering phenomena existed in the traditional sliding mode algorithm.

\section{Theory of T-S fuzzy model}

A T-S fuzzy model can express a nonlinear discrete system with $n$ rules:

$R^{i}$ : If $x_{1}$ is $A_{1}^{i}$ and $\ldots$ and $x_{m}$ is $A_{m}^{i}$, then

$$
y^{i}=p_{0}^{i}+p_{1}^{i} x_{1}+p_{2}^{i} x_{2}+\ldots+p_{m}^{i} x_{m},
$$

where $x_{1}=y(k-1), \ldots, x_{v}=y(k-v), x_{v+1}=u(k-1), \ldots$, $x_{m}=u(k-l), m=v+l, A^{i}$ is the fuzzy set, $p_{j}^{i}$ is constant, where $i=1, \ldots, n, j=1, \ldots, m$.

Given a generalized input vector $\left(x_{1}, x_{2}, \ldots, x_{m}\right)$ and the weighted average of $y^{i}(i=1,2, \ldots, n)$, the output of T-S fuzzy system at any time is computed as follows:

$$
y(x)=\frac{\sum_{j=1}^{m} y^{i}\left(\prod_{i=1}^{n} \mu_{A_{i}^{j}}\left(x_{i}\right)\right)}{\sum_{j=1}^{m}\left(\prod_{i=1}^{n} \mu_{A_{i}^{j}}\left(x_{i}\right)\right)} .
$$

Rewrite the equation (4) and it yields

$$
y(x)=L^{T} \xi(x),
$$

where $L=\left[y^{1} \ldots y^{m}\right]^{T}, \xi(x)=\left[\xi^{1}(x) \ldots \xi^{m}(x)\right]^{T}$.

$$
\xi(x)=\frac{\prod_{i=1}^{n} \mu_{A_{i}^{j}}\left(x_{i}\right)}{\sum_{j=1}^{m}\left(\prod_{i=1}^{n} \mu_{A_{i}^{j}}\left(x_{i}\right)\right)}
$$

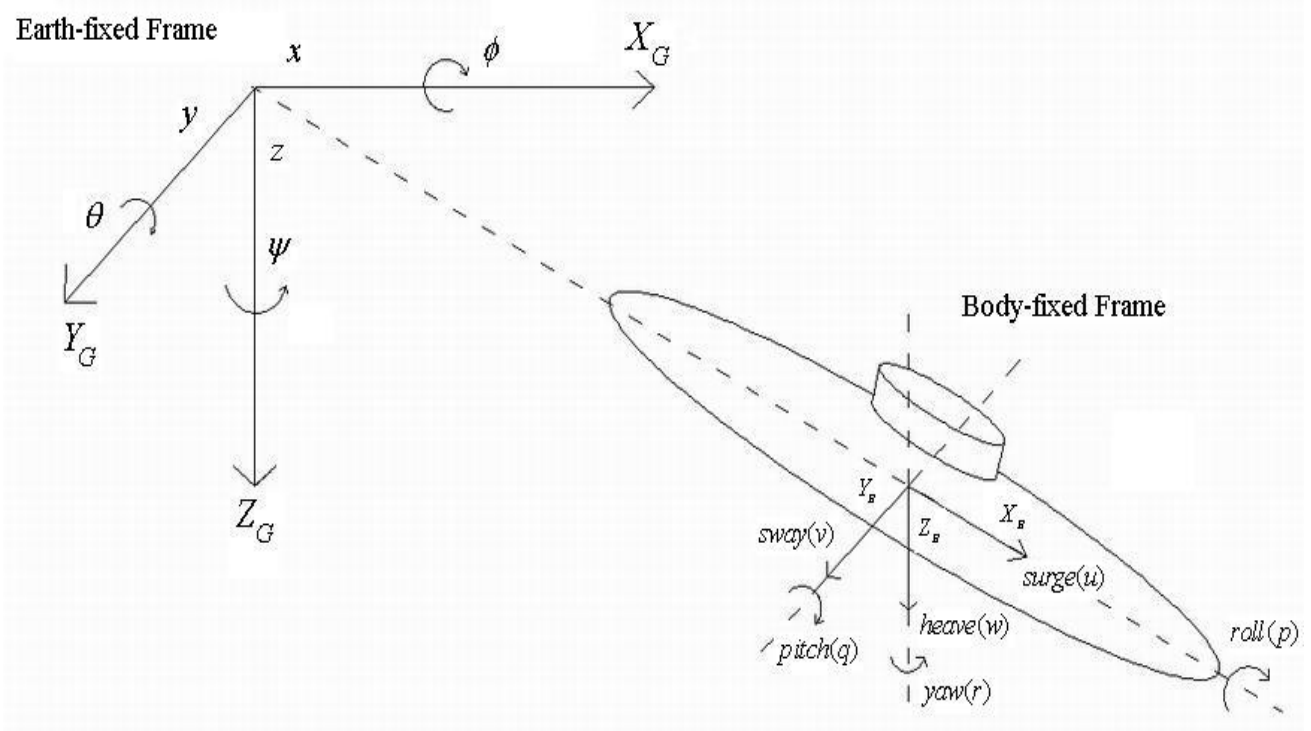

Fig. 1. Frames and variables of underwater robot 


\section{Adaptive controller design}

In this section, a new adaptive controller design is presented. The output controller design is assumed a tracking problem. Thus, a control law can be generated, when the state $\eta$ tracks the desired command $\eta_{d}$ and the tracking error vector $e(t)=\eta_{d}-\eta$ is defined. Moreover, the sliding surface following the convenient design form is presented as

$$
S(t)=(d / d t+\lambda)^{2} \int_{0}^{\tau} e(\tau) d t
$$

where $S \in R^{6}$ and $\lambda$ is a constant positive definite matrix.

Here, a new control vector $U(t)=M_{\eta}^{-1}(\eta) \tau$ is defined. Following the common steps of locating the sliding surface, the equivalent control law $U_{e q}(t)$ can be derived when

$$
\left.\dot{S}(t)\right|_{U}=U_{e q}=0
$$

For tracking the control problem, $U(t)$ is designed with respect to the state remaining on the $S$ surface $(S(t)=0$ if $t>0)$.

Following the equation (1), (7), and (8), the equivalent control law can be calculated as

$$
\begin{aligned}
U_{e q}= & \ddot{\eta}_{d}+M_{\eta}^{-1}(\eta)\left[C_{\eta}(\eta, \dot{\eta}) \dot{\eta}+D(\eta, \dot{\eta}) \dot{\eta}+\right. \\
& \left.g_{\eta}(\eta)+\hat{\delta}\right]+2 \lambda \dot{e}(t)+\lambda^{2} e(t)
\end{aligned}
$$

where $\hat{\delta}$ is the estimate of disturbance.

Generally, the disturbance $\delta$ is unknown and time varying. In order to ensure the feasibilities of the controller, the unknown disturbance $\delta$ is bounded. Thus, an adaptive fuzzy model can be proposed to estimate the unknown disturbance $\delta$.

Following the T-S fuzzy model theory, a zero order TSK (Takagi-Sugeno-Kang) is used. Considering each output from each fuzzy rule, the output $\hat{\delta}$ is described as follows:

$$
\hat{\delta}(\eta)=\frac{\sum_{r=1}^{N} \omega_{r} \cdot \hat{\delta}_{r}}{\sum_{r=1}^{N} \omega_{r}}
$$

$$
\hat{\delta}(\eta)=\hat{L}_{\delta}^{T} \psi(\eta)
$$

Here, $L_{\delta}=\left[L_{\delta 1}, L_{\delta 2}, \ldots ., L_{\delta N}\right]^{T}$ is the eigenvalue vector $L_{d}$, which includes $d$ from each rule

$$
\psi(\eta)=\left[\psi_{1}(\eta), \psi_{2}(\eta), \ldots, \psi_{N}(\eta)\right]^{T},
$$

where $\psi_{N}(\eta)=\omega_{r} / \sum_{r=1}^{N} \omega_{r}$ and $\omega_{r}$ is the length of each rule. The adaptive approximation parameters are given

$$
\dot{L}_{\delta}=-K_{1} S \psi(\eta)
$$

where $K_{1}$ is the positive constant to adaptive velocity.

Like the adaptive fuzzy model definition of disturbance $\delta$, an adaptive fuzzy smoother is presented for the discontinuous terms of the sliding mode control. The switch control law in sliding mode is given as follows:

$$
U_{s w}=h(S) .
$$

The output of fuzzy model can be presented as:

$$
h(S)=L_{h}^{T} \phi(S) \text {. }
$$

The adaptive parameter $L_{h}$ can be calculated as:

$$
\dot{L}_{h}=-K_{2} S \phi(S) \text {, }
$$

where $K_{2}$ is the positive constant. Therefore, this new adaptive fuzzy controller with prediction and smoother (AFPS) can be presented as:

$$
\begin{aligned}
U(t)= & \ddot{\eta}_{d}+M_{\eta}^{-1}(\eta)\left[C_{\eta}(\eta, \dot{\eta}) \dot{\eta}+D(\eta, \dot{\eta}) \dot{\eta}+g_{\eta}(\eta)\right. \\
& +\hat{\delta}]+2 \lambda \dot{e}(t)+\lambda^{2} e(t)+h(S)
\end{aligned}
$$

Theorem 1. When the underwater robot uses the controller (equation (16)), the system can enter the sliding surface $S(t)$ and keep stable.

Proof: Given Lyapunov function $V$

$$
V=\frac{1}{2} S^{2}+\frac{1}{2 M_{\eta}(\eta) K_{1}} \theta_{\theta}^{T} \theta_{\delta}+\frac{1}{2 K_{2}} \theta_{h}^{T} \theta_{h},
$$

where $\theta_{\delta}=L_{\delta}^{*}-L_{\delta}, \theta_{h}=L_{h}^{*}-L_{h}$ and $\hat{L}^{*}$ is the optimal parameter vector associated with the optimal estimates $\hat{\delta}^{*}$ and $h^{*}$.

Let the optimal parameters as

$$
L_{\delta}^{*}=\arg \min _{L_{\delta} \in \Omega_{\delta}}[\sup |\hat{\delta}-\delta|],
$$




$$
L_{h}^{*}=\arg \min _{L_{h} \in \Omega_{h}}[\sup |\hat{h}-h|],
$$

where $\Omega_{\delta}, \Omega_{h}$ are respectively the sets of $L_{\delta}$ and $L_{h}$.

Let the minimal approximation error $\varepsilon$ as

$$
\begin{aligned}
& \varepsilon=\delta-\delta^{*} \text { and }|\varepsilon| \leq \varepsilon_{\max } \\
& \dot{V}= S \dot{S}+\frac{1}{M_{\eta}(\eta) K_{1}} \theta_{\delta}^{T} \dot{\theta}_{\delta}+\frac{1}{K_{2}} \theta_{h}^{T} \dot{\theta}_{h} \\
&= S\left(\ddot{\eta}_{d}-U(t)+M_{\eta}^{-1}\left[C_{\eta}(\eta, \dot{\eta}) \dot{\eta}+D(\eta, \dot{\eta}) \dot{\eta}+\right.\right. \\
&\left.g_{\eta}(\eta)+\delta(\eta)\right]+2 \lambda \dot{e}(t)+ \\
&\left.\lambda^{2} e(t)\right)+\frac{1}{M_{\eta}(\eta) K_{1}} \theta_{\delta}^{T} \dot{\theta}_{\delta}+\frac{1}{K_{2}} \theta_{h}^{T} \dot{\theta}_{h} \\
&= S M_{\eta}^{-1}\left[\left(\delta(\eta)-\hat{\delta}^{*}(\eta)\right)+\left(\hat{\delta}^{*}(\eta)-\hat{\delta}(\eta)\right)\right] \\
&-S h(S)+\frac{1}{M_{\eta}(\eta) K_{1}} \theta_{\delta}^{T} \dot{\theta}_{\delta}+\frac{1}{K_{2}} \theta_{h}^{T} \dot{\theta}_{h} \\
&= S M_{\eta}^{-1}\left[\varepsilon+\left(L_{\delta}^{*}-L_{\delta}\right)^{T} \psi(\eta)\right]-S h(S) \\
&+\frac{1}{M_{\eta}(\eta) K_{1}} \theta_{\delta}^{T} \dot{\theta}_{\delta}+\frac{1}{K_{2}} \theta_{h}^{T} \dot{\theta}_{h} \\
&= S M_{\eta}^{-1}\left[\varepsilon+\theta_{\delta}^{T} \psi(\eta)\right]-S h(S)+\frac{1}{M_{\eta}(\eta) K_{1}} \theta_{\delta}^{T} \dot{\theta}_{\delta} \\
&= S M_{\eta}^{-1} \varepsilon+\frac{1}{K_{2}} \theta_{h}^{T} \dot{\theta}_{h}+S\left(h^{*}(S)-h(S)\right)-S h^{*}(S) \\
&+\frac{1}{K_{2}} \theta_{h}^{T} \dot{\theta}_{h} \\
&= S M_{\eta}^{-1} \varepsilon+\frac{\theta_{\delta}^{T}}{M_{\eta} K_{1}}\left[S K_{1} \psi(\eta)+\dot{\theta}_{\delta}\right]+\frac{1}{K_{2}} \theta_{h}^{T} \dot{\theta}_{h}-S h(S) \\
& S^{*}(S) h(S)-S h^{*}(S) \\
& h^{*}(S)=\varepsilon^{2}(S) \\
&
\end{aligned}
$$

Let $h^{*}(S)=\varepsilon_{\Delta} \operatorname{sgn}(S)$, where $\varepsilon_{\Delta} \geq 0$.

$$
\begin{aligned}
& \dot{V}=S M_{\eta}^{-1} \varepsilon+S \theta_{h}^{T} \phi(S)+\frac{1}{K_{2}} \theta_{h}^{T} \dot{\theta}_{h}-S h^{*}(S) \\
& =S M_{\eta}^{-1} \varepsilon+\frac{\theta_{h}^{T}}{K_{2}}\left(K_{2} S \phi(S)+\dot{\theta}_{h}\right)-S h^{*}(S)
\end{aligned}
$$

$\leq S \varepsilon_{m}-\varepsilon_{\Delta}|S|$

where $S$ is bounded. According to the fuzzy approach theory, the adaptive fuzzy controller can make the error $\varepsilon_{m}$ very small to keep $\dot{V} \leq 0$. Then, $V(t) \leq V(0)$ can be satisfied. The theorem can be proven.

\section{Simulations and Experiments}

\subsection{Simulations}

The precise control and navigation of underwater robot can hardly be realized with limited kinetic knowledge and complex application environment. Therefore, the system simulation of the dynamic model and control system kinematics are helpful for the underwater robot. Generally, the parameters of dynamic equation are nonlinear, coupled, time-varying, and unstable. It is difficult to obtain accurate hydrodynamic equations. On the other hand, due to the disturbance of flow, waves etc., it is also difficult to obtain the accurate disturbance model. Thus, the controller should have the strong robustness to the uncertainties, disturbance etc.

In this paper, a large time-varying flow disturbance is considered and simulations are carried out between the new control method AFPS and the sliding mode controller (SMC) with smooth control. SMC controller is presented ${ }^{17}$ and the parameters are defined as:

$$
\lambda=0.75, \rho=9.2 \text {. }
$$

In AFPS simulations, the parameters are listed as follows:

$$
\begin{aligned}
& M_{0}=\operatorname{diag}\{112.0,124.0,117.0,12.5\} ; \\
& D_{0}=\operatorname{diag}(118|u|, 156|v|, 178|w|, 9.18|r|) ; \\
& g=\left[\begin{array}{llll}
0 & 0 & 0 & 0
\end{array}\right] ; K_{1}=12 ; K_{2}=3.6 ; \lambda=1.3 ;
\end{aligned}
$$

The matrix $C$ is simplified, due to the lack of knowledge concerning the values of its elements ${ }^{13}$. It is given as: 

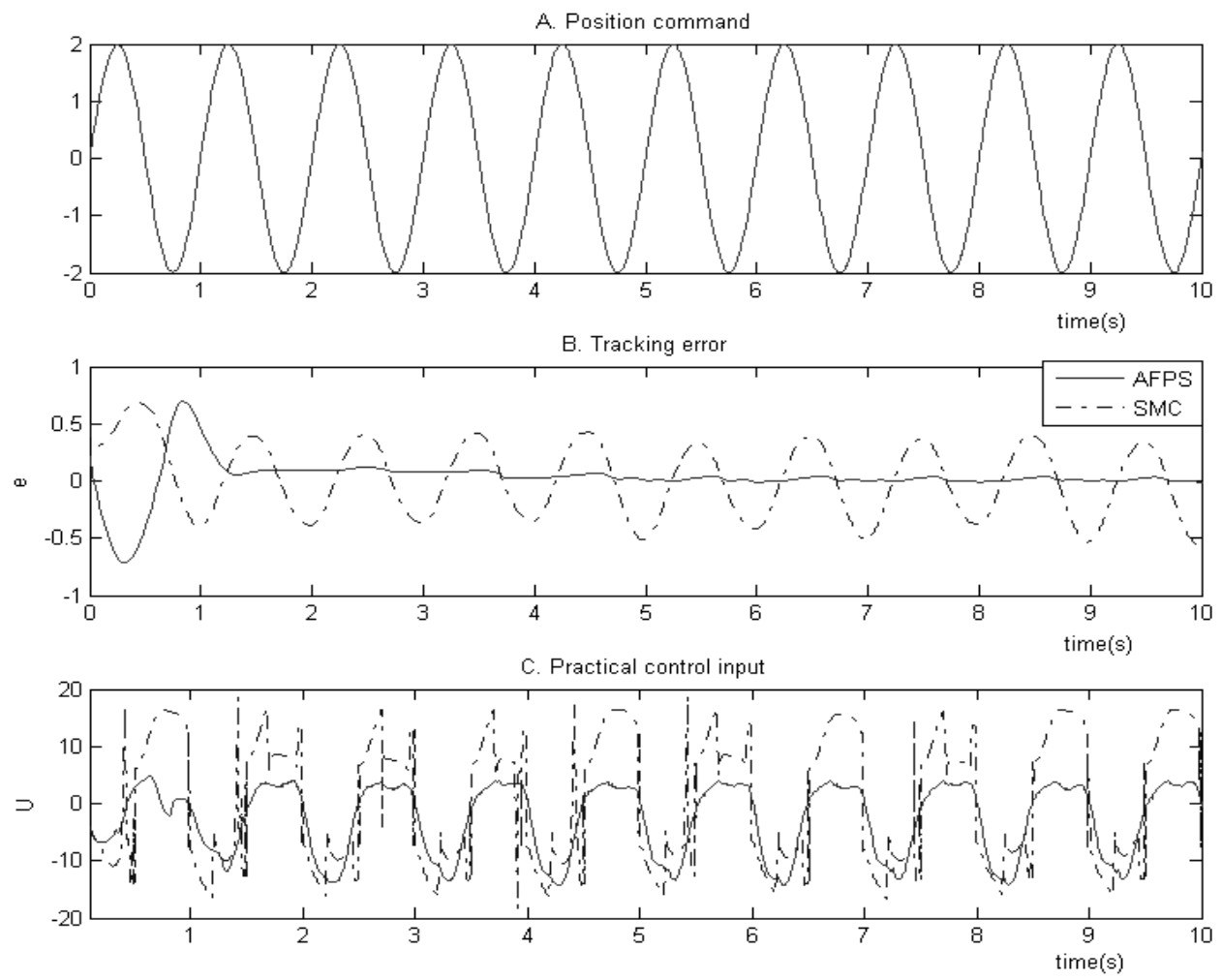

Fig. 2. Simulation results

$$
C=\left[\begin{array}{cccc}
0 & 0 & 0 & 118 v \\
0 & 0 & 0 & -114 u \\
0 & 0 & 0 & 0 \\
-118 v & -114 u & 0 & 0
\end{array}\right] .
$$

The command of yaw motion trajectory is

$$
\psi(t)=2 \sin (2 \pi t)
$$

The time-varying large flow disturbance model is given by

$$
\begin{aligned}
\delta=[ & \sin (7 \pi t)+\sin (4 \pi t)+\sin (3 \pi t)+\sin (9 \pi t)+1 \\
& \sin (7 \pi t)+\sin (4 \pi t)+\sin (3 \pi t)+\sin (9 \pi t)+1 \\
& \sin (7 \pi t)+\sin (4 \pi t)+\sin (3 \pi t)+\sin (9 \pi t)+1 \\
& \sin (7 \pi t)+\sin (4 \pi t)+\sin (3 \pi t)+\sin (9 \pi t)+1]^{T} \\
& * M * 0.8 .
\end{aligned} .
$$

The membership functions of disturbance are depicted as the following:

$$
\begin{aligned}
& \mu_{\delta^{1}}\left(x_{i}\right)=\exp \left[-\left(0.8 * x_{i}+\pi / 5\right) /(\pi / 25)^{2}\right], \\
& \mu_{\delta^{2}}\left(x_{i}\right)=\exp \left[-\left(0.8 * x_{i}+\pi / 10\right) /(\pi / 25)^{2}\right], \\
& \mu_{\delta^{3}}\left(x_{i}\right)=\exp \left[-\left(0.8 * x_{i} /(\pi / 24)^{2}\right],\right. \\
& \mu_{\delta^{4}}\left(x_{i}\right)=\exp \left[-\left(0.8 * x_{i}-\pi / 10\right) /(\pi / 25)^{2}\right], \\
& \mu_{\delta^{5}}\left(x_{i}\right)=\exp \left[-\left(0.8 * x_{i}-\pi / 5\right) /(\pi / 25)^{2}\right] .
\end{aligned}
$$

Define the membership functions of switch control law as follows:

$$
\begin{aligned}
& \mu_{S^{1}}(S)=\frac{1}{1+\exp [0.9 * 5(S+4)]}, \\
& \mu_{S^{2}}(S)=\exp \left[-(0.9 * S+0.8)^{2}\right], \\
& \mu_{S^{3}}(S)=\exp \left[-0.9 * S^{2}\right], \\
& \mu_{S^{4}}(S)=\exp \left(-(0.9 * S-0.8)^{2}\right), \\
& \mu_{S^{5}}(S)=\frac{1}{1+\exp [5(0.9 * S-4)]} .
\end{aligned}
$$

The vector of adjustable parameters was initialized with zero values, such as $\hat{L}=0$. The simulation results 
are shown in Fig. 2. Fig. 3 shows the comparisons between the disturbance prediction and actual disturbance. Although the prediction can hardly track the exact the disturbance, the trend and the varieties can be almost

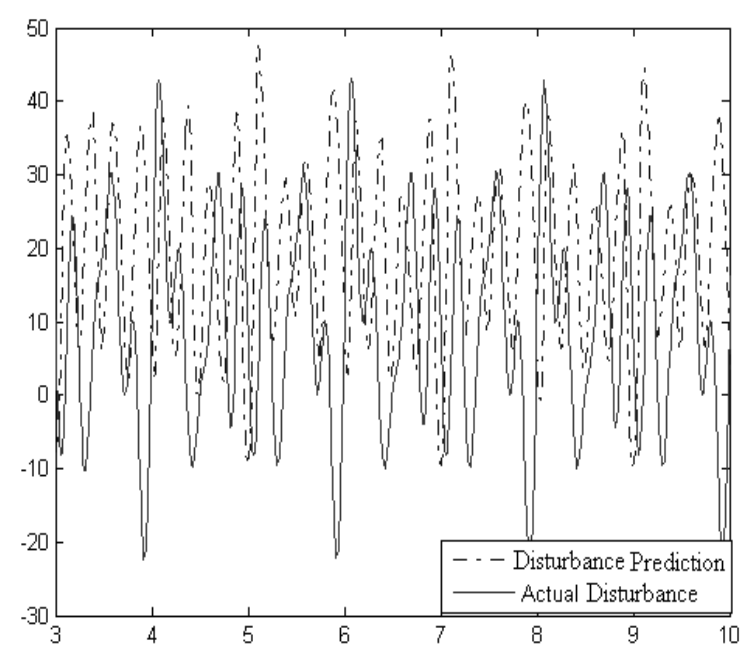

Fig. 3.Comparisons between disturbance prediction and actual disturbance

grasped. The part A of Fig. 2 shows the command trajectory of yaw motion. Part B shows tracking errors of two control algorithms. Obviously, the new AFPS algorithm can converge fast and the residual errors can be largely reduced compared with SMC method with smooth control. Meanwhile, from part C, the practical control input of AFPS show much smoother characters and the chattering errors decrease a lot.

Through the aid of these adaptive disturbance predictions, the compensation model can be estimated. AFPS algorithm applies this compensation model to the controller. From these results, this new adaptive fuzzy model plays an important part in this controller.

In this simulation, a time-varying and large disturbance is considered. These two controllers both show the robust abilities and make the system stable after regulations. However, SMC method with smooth control shows its deficiencies i.e. larger errors, long time of convergence, and the unstable chattering phenomenon is existed in its control input and the vibration amplitudes are larger than AFPS. Therefore, AFPS show better tracking results and improved control performance from the simulation results.

\subsection{Experiments}

To verify the effectiveness shown in the simulation results, yaw control experiments were implemented in the basin by small underwater robot. These experiments have considered the nonlinear time varying flow disturbance. The size of underwater robot is $31 \mathrm{~cm} * 22.5 \mathrm{~cm} * 21 \mathrm{~cm}$; the weight in the air is $60 \mathrm{~kg}$; the max navigation speed is $2.6 \mathrm{knot}$; the max diving depth is $152 \mathrm{~cm}$. Fig. 4. shows the experiment scene of underwater robot. In the

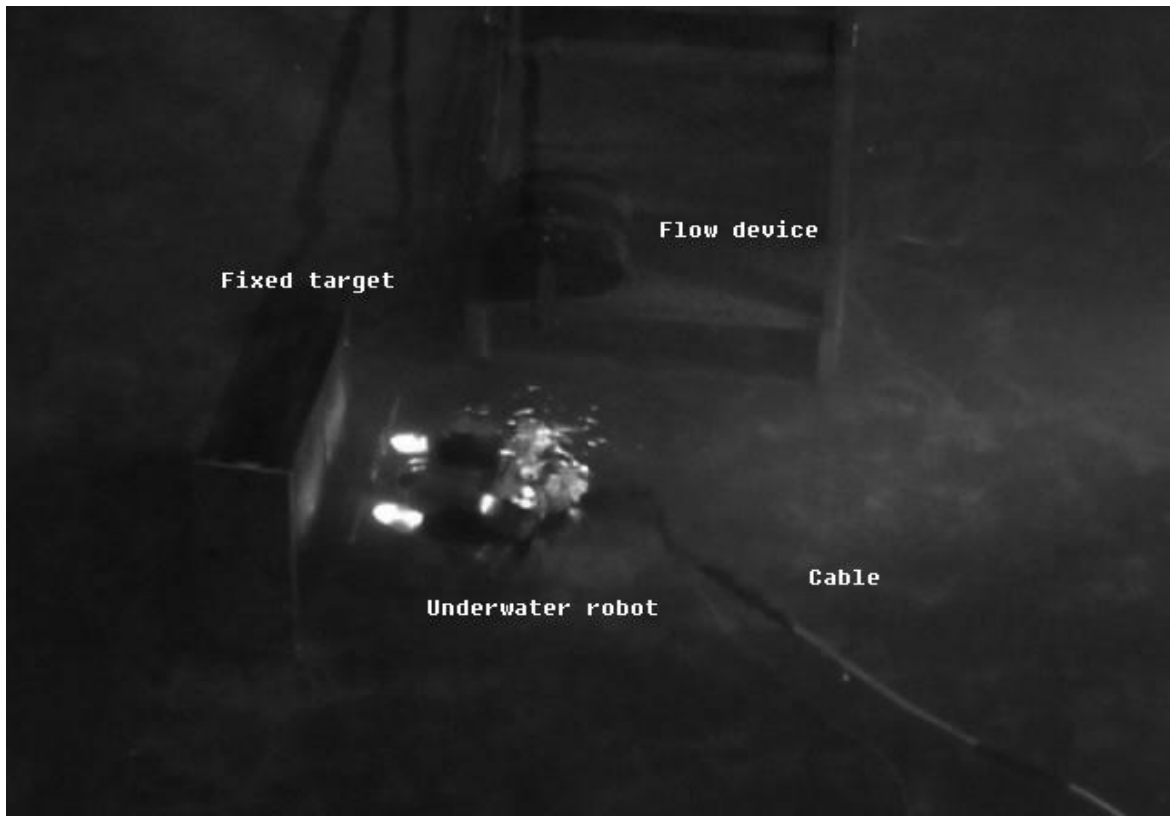

Fig. 4. Experiment of underwater robot 

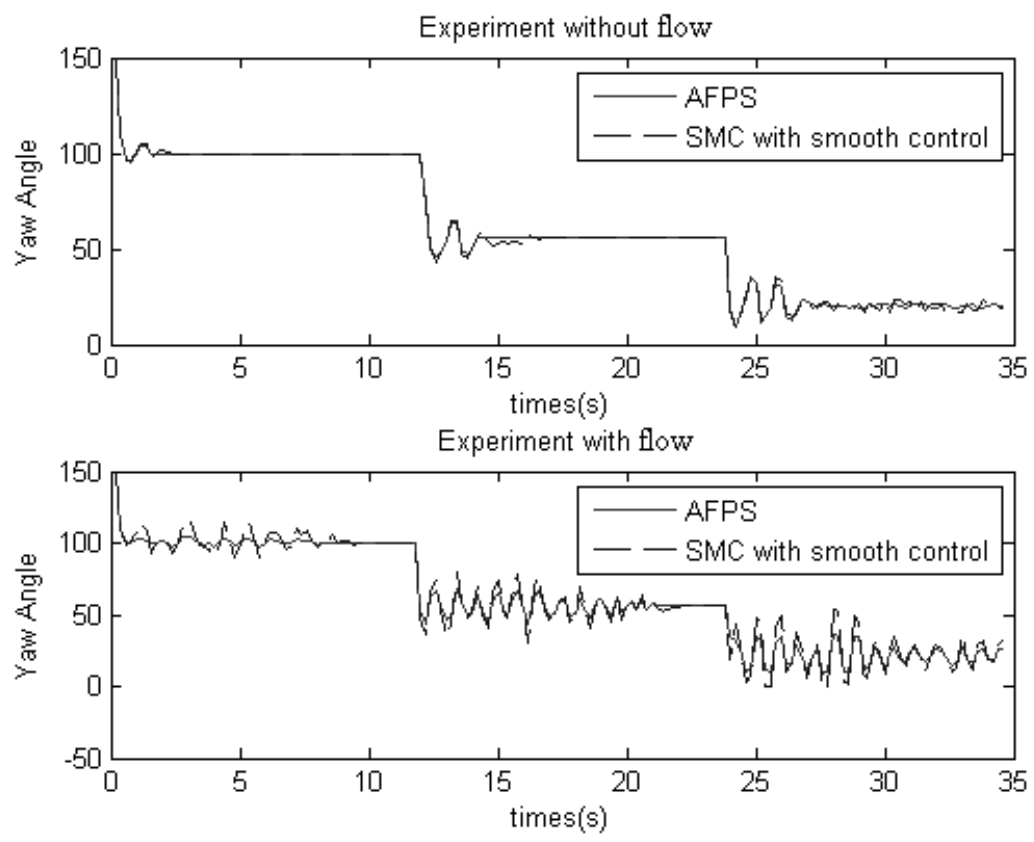

Fig. 5. Controller results comparison between AFPS and SMC with smooth control

experiment, the yaw angle command is changed from 100 degrees $\rightarrow 56$ degrees $\rightarrow 20$ degrees.

Both control algorithms are applied to the on-line practical control experiments. These comparisons consisted of two situations. One chooses static water and the other one considers the flow disturbance. The timevarying flow speed is defined as $V_{\text {Drift }}=0.15 *(\sin (4 \pi t)+1) \mathrm{m} / \mathrm{s}$ and the wave disturbance is ignored. The control parameters are similar to the simulations with small changes.

Therefore, the experiment results of these two situations can be collected and shown in Fig.5. Table 1 shows detailed information including the errors and stable times. The results from both situations show that both controllers can reach relatively steady status. The stable time increases when the command angle reduces. For the first two degrees (100 degrees and 56 degrees), the yaw angle can become steady at the command angle after adjustment. However, at 20 degrees, the underwater robot cannot reach the constant command angle. It swings in a relative range and becomes relatively steady status. Compared with results without flow, the results with flow show larger angle errors and the stable times become longer.

Due to the small weight of this underwater robot, the disturbance forces from the cable become relatively large and can make troubles to the control performance. When the command angle is reduced, the front part of underwater robot moves close to the cable. The disturbance forces from the cable grow large that cause the controller beyond the regulation range and hardly reach the ideal steady status i.e. at 20 degrees.

Though there are some deficiencies, the controllers remain well characters to help the underwater robot to keep the relative steady movements. From the experimental results, it is obviously that the new proposed AFPS algorithms show large improvement compared with SMC with smooth control. The errors are smaller and the stable times are shorter in all experiment situations. Although AFPS cannot keep ideal steady status at 20 degrees like SMC with smooth control, the errors of AFPS are much smaller and the underwater robot keeps a very small swing movement which can be acceptable for the practical applications. The control input of AFPS in the experiments also show much better smoothness and the chattering phenomena is largely reduced. These experiment results correspond with the simulation results. Thus, AFPS shows better robustness and the results can demonstrate the performance and effectiveness of this new control algorithm.

For the shortcoming existed in the current works, a mathematical model of the cable force is important to 
Table 1. Results statistics

\begin{tabular}{|c|c|c|c|c|}
\hline \multicolumn{5}{|c|}{ AFPS } \\
\hline & \multicolumn{2}{|c|}{ Without flow } & \multicolumn{2}{|c|}{ With flow } \\
\hline Command angle & Errors (angle) & Stable time & Errors (angle) & Stable time \\
\hline 100 & 7.7460 & 1.6 & 12.4861 & 8.1 \\
\hline 56 & 29.5973 & 2.3 & 43.1626 & 9.2 \\
\hline 20 & 32.1723 & no & 60.0546 & no \\
\hline \multicolumn{5}{|c|}{ SMC with smooth control } \\
\hline & \multicolumn{2}{|c|}{ Without flow } & \multicolumn{2}{|c|}{ With flow } \\
\hline Command angle & Errors (angle) & Stable time & Errors (angle) & Stable time \\
\hline 100 & 9.0357 & 2.4 & 67.3571 & 9.8 \\
\hline 56 & 32.7218 & 4.8 & 73.4301 & 12 \\
\hline 20 & 36.0632 & no & 116.0495 & no \\
\hline
\end{tabular}

improve the control abilities and the control design for solving more complex disturbance is also necessary to be investigated in future studies.

\section{Conclusion}

In this paper, a new adaptive controller AFPS is constructed by adopting adaptive T-S fuzzy algorithm and sliding mode control algorithm. Based on the adaptive T-S fuzzy model, a disturbance prediction and a control smoother are proposed for the trajectory regulation of underwater robot. Using the new adaptive fuzzy model, the uncertainties, and disturbance can be estimated. The chattering phenomena are largely reduced by adaptive nonlinear smoother. The stability and convergence properties of this new controller are proven by Lyapunov stability theory. The simulation results considering a large time-varying and nonlinear flow disturbance proves the performance of this new controller. Though there are some deficiencies shown in the experimental studies, AFPS remains better characters i.e. smaller errors, shorter convergence time compared with SMC with smooth control. The experimental results in different environment also demonstrate that AFPS has better robustness and improved abilities for the practical applications.

\section{Acknowledgements}

This work was partially supported by the National Natural Science Foundation of China (Grant No: 50779033) and National 863 High-Tech R \& D Program of China (Grant No: 2007AA11Z250).
This work was also partly supported by the Region Rhône-Alpes of France under the project Mira Recherche 2008, and the joint project of French ANR 2009 (under ANR-09-BLAN-0372-01) and Chinese NSFC (under 30911130364).

\section{References}

1. J. Yuh, Modeling and control of underwater robot vehicle, IEEE T. Syst. Man Cyb. 20(6) (1990) 1475-1483.

2. J. Guo, F. C. Chiu, and C. C. Huang, Design of a sliding mode fuzzy controller for the guidance and control of an autonomous underwater vehicle, Ocean Eng. 30(16) (2003) 2137-2155

3. A. Pisano, E.Usai, Output-feedback control of an underwater vehicle prototype by higher-order sliding modes, Automatica. 40(9) (2004) 1525-1531.

4. X. Song, J. W. Ye and L. M. Wu, Application of the integral sliding mode controller with fuzzy logic to submersible vehicle, Int. J. Innov. Comput. Inf. Contro. 3(4) (2007) 897-906.

5. X. Song, Z. J. Zou and J. W. Ye, Modeling, design and experiment of improved super-mini underwater robot, Int. J. Innov. Comput. Inf. Contro. 7(3) (2011) 1109-1119.

6. C. Cavtat, A comparison of classical, neural and fuzzy control for an underwater vehicle, in Proceedings of the 7th WSEAS international conference on Neural networks, (Croatia, 2006), pp.61-66.

7. S. Alavandar, T. Jain, M. J. Nigam, Bacterial foraging optimized hybrid fuzzy precompensated PD control of two link rigid-flexible manipulator, Int. J. Comput. Intell. Syst. 2(1) (2009) 51-59.

8. S. Sentürk, Fuzzy regression control chart based on alfacut approximation, Int. J. Comput. Intell. Syst. 3(1) (2010) 123-140. 
9. L. K. Wong, F. H. F. Leung, and P. K. S. Tam, A fuzzy sliding controller for nonlinear systems, IEEE T. Ind. Electron. 48(1) (2001) 32-37.

10. K. Taghizadeh, M. Bagherpour, I. Mahdavi, Application of fuzzy multi-objective linear programming model in a multi-period multi-product production planning problem, Int. J. Comput. Intell. Syst. 4(2) (2011) 228-243.

11. W. M. Bessa, M. S. Dutra, and E. Kreuzer, Thruster dynamics compensation for the positioning of underwater robotic vehicles through a fuzzy sliding mode based approach, in COBEM 2005-Proceedings of the 18th International congress of mechanical engineering, (Ouro Preto, Brasil, 2005).

12. W. M. Bessa, M. S. Dutra, and E. Kreuzer, Depth control of remotely operated underwater vehicles using an adaptive fuzzy sliding mode controller, Robot. Auton. Syst. 56(8) (2008) 670-677.

13. T. Z. Wu and Y. T. Juang, Adaptive fuzzy sliding-mode controller of uncertain nonlinear systems, ISA Trans. 47(3) (2008) 279-85.

14. C. W. Tao, M. L. Chan and T. T. Lee, Adaptive fuzzy sliding mode controller for linear systems with mismatched time-varying uncertainties, IEEE Trans. Syst. Man. Cybern. B Cybern. 33(2) (2003) 283-94.

15. T. I. Fossen, Guidance and control of ocean vehicles, (Wiley, New York, 1994).

16. D. A. Smallwood, and L. L. Whitecomb, Model-based dynamic positioning of underwater robotic vehicles: theory and experiments, IEEE J. Ocean Eng. 29(1) (2004) 169186.

17. T. Chatchanayuenyong, M. Parnickkun, Neural network based-time optimal sliding mode control for an autonomous underwater robot, Mechatronics. 16(8) (2005) 471-478. 